\title{
A RECURRENT 1992delCT MUTATION OF THE TYPE X COLLAGEN GENE IN A JAPANESE PATIENT WITH SCHMID METAPHYSEAL CHONDRODYSPLASIA
}

\author{
Yoshito MatsuI, ${ }^{1, *}$ Tomoatsu KImura, ${ }^{2}$ Noriyuki TsumaKI, ${ }^{1}$ \\ Natsuo YASUI, ${ }^{3}$ and Takahiro OCHI ${ }^{1}$ \\ ${ }^{1}$ Department of Orthopaedic Surgery, Osaka University Medical School, \\ 2-2 Yamadaoka, Suita 565, Japan \\ ${ }^{2}$ Department of Orthopaedic Surgery, Osaka Rosai Hospital, \\ 1179-3 Nagasone-cho, Sakai 591, Japan \\ ${ }^{3}$ Department of Orthopaedic Surgery, Osaka Medical Center and \\ Research Institute for Maternal and Child Health, 840 Murodo-cho, Izumi 590-02, Japan
}

Summary We report here a recurrent frameshift mutation within the carboxyl-terminal noncollagenous domain coding region of the type $\mathrm{X}$ collagen gene (COL10A1) in a Japanese patient with Schmid metaphyseal chondrodysplasia. The mutation involves deletion of a CT dinucleotide from position 1992 (1992delCT), and produces a frameshift which creates a premature termination codon close to the site of the deletion. The predicted length of the mutant polypeptide is 664 amino acids, which is shorter than the wild type polypeptide (680 amino acids). A 1992delCT mutation of COL10A1 has been previously reported in one family. The independent occurrence of de novo mutation of this specific dinucleotide repeat suggests that this region is a possible mutational hot spot on COL1OA1.

Key Words Schmid metaphyseal chondrodysplasia (SMCD), mutation, type $\mathrm{X}$ collagen gene (COL1OAl), Japanese population, heteroduplex analysis

Schmid metaphyseal chondrodysplasia (SMCD) is an autosomal dominant disorder characterized by mild dwarfism and coxa vara. Seventeen different SMCD mutations reported to date have all been located within the carboxyl (C)-terminal noncollagenous domain coding region of the type $\mathrm{X}$ collagen gene (COL10A1) (Warman et al., 1993; Wallis et al., 1994; McIntosh et al., 1994, 1995; Dharmavar-

Received March 21, 1996; Revised version accepted June 17, 1996.

${ }^{*}$ To whom correspondence should be addressed. 
am et al., 1994; Chan et al., 1995; Bonaventure et al., 1995). It has been suggested that mutations of this domain prevent the mutant polypeptides from participation in stable triple-helix formation and lead to a reduction in the amount of normal protein. Alternatively, the presence of abnormal type $\mathrm{X}$ homotrimers may interfere with the formation of large multimeric aggregates. We report here a recurrent frameshift mutation within the C-terminal noncollagenous domain coding region of COL10A1 in a Japanese patient with SMCD.

A 3-year-old Japanese girl was diagnosed as having SMCD on the basis of clinical features and radiographic data at the Osaka Medical Center and Research Institute for Maternal and Child Health. Her parents were clinically normal and were unrelated. DNA was extracted from peripheral blood leukocytes by a standard method (Sambrook et al., 1989). Since all SMCD mutations reported to date have been located within the C-terminal noncollagenous domain of COL10A1, we used two sets of polymerase chain reaction (PCR) primers to study the portion of the genomic DNA that included a sequence encoding this entire C-terminal domain. The sequences of the primers used (primer H15: 5'-TCAATGGACCCACCGGGC-3', primer H18: 5'-TGATGGCACTCCCTGAAGCCTG-3', primer H17: 5'-GCCTGTGTCTGCTTTTACTG-3', and primer H23: 5'-TTCAGCCTACCTCCATATGC-3') were identical to those reported previously (McIntosh et al., 1994). Primers H15 and H18 amplified a 471-bp product, while primers H17 and $\mathrm{H} 23$ amplified a 490-bp product. Amplification was performed with $0.5 \mathrm{U}$ of TaKaRa Taq (Takara, Shiga, Japan) in the following reaction mixture with a total volume of $25 \mu \mathrm{l}: 50 \mathrm{mM} \mathrm{KCl}, 1.5 \mathrm{mM} \mathrm{MgCl}_{2}, 10 \mathrm{mM}$ Tris- $\mathrm{HCl}$ ( $\mathrm{pH} \mathrm{8.3),} 100 \mu \mathrm{M}$ of each dNTP, $100 \mathrm{ng}$ DNA, and 12.5 pmol of each primer. Thirty cycles of $30 \mathrm{sec}$ each at $94^{\circ} \mathrm{C}, 62^{\circ} \mathrm{C}$, and $72^{\circ} \mathrm{C}$ were used. The PCR products were identified on $3 \%$ low melting point agarose gel and subjected to heteroduplex analysis using MDE $^{\text {TM }}$ gel (AT Biochem, Malvern, PA) according to the manufacturer's instructions. In addition, the amplified products were purified with a QIAEX II gel extraction kit (Qiagen, Chatsworth, CA) and directly sequenced using a Taq Dye Terminator cycle sequencing kit (Applied Biosystems, Foster City, CA) and an Applied Biosystems 373A DNA sequencer.

MDE $^{\mathrm{TM}}$ gel electrophoresis of the PCR products amplified with primers $\mathrm{H} 17$ and $\mathrm{H} 23$ indicated the presence of heteroduplex bands in the proband, but not in her parents (Fig. 1). Direct DNA sequencing of the PCR product suggested that the patient was heterozygous for the deletion of a CT dinucleotide from position 1992 (1992delCT) (data not shown). In order to confirm this mutation, the PCR product of primers $\mathrm{H} 17$ and $\mathrm{H} 23$ from the patient was digested with HindIIJ/NsiI and was subcloned into the HindIII/Pst I cloning sites of pBluescript II SK ${ }^{+}$ (Stratagene, La Jolla, CA). The presence of $1992 \mathrm{delCT}$ mutation was confirmed by sequencing of DNA from individual subclones (Fig. 2). This frameshift mutation created a premature termination codon close to the site of the deletion. The predicted length of the mutant polypeptide is 664 amino acids, which is shorter 


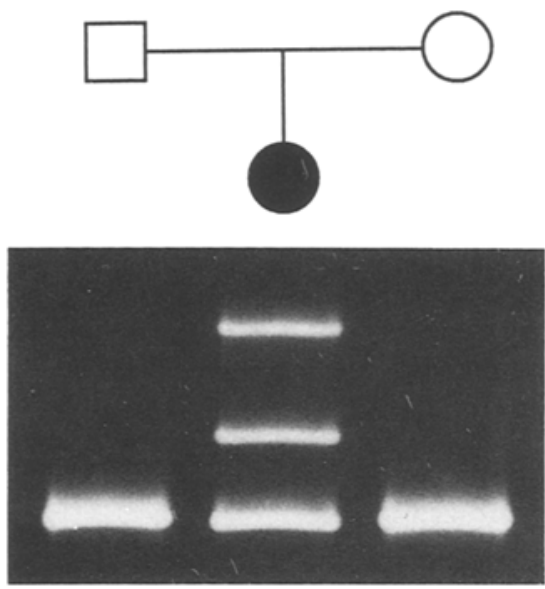

Fig. 1. Heteroduplex analysis of PCR products amplified with primers $\mathrm{H} 17$ and $\mathrm{H} 23$ from the SMCD patient and her parents. Note the additional heteroduplex bands of the SMCD patient, but not parents.
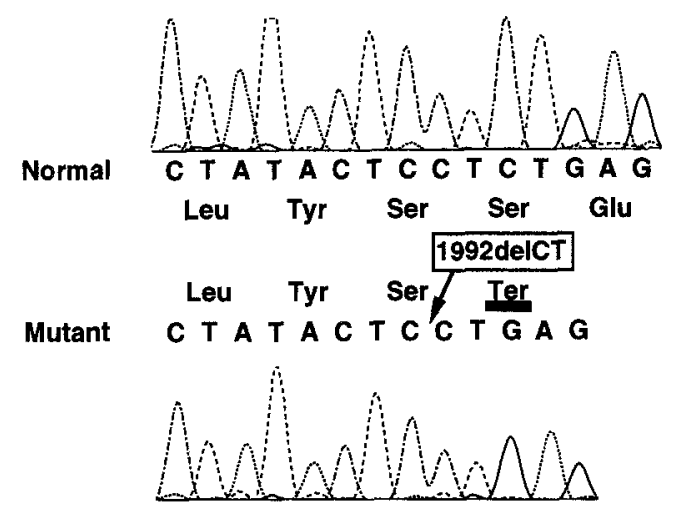

Fig. 2. DNA sequencing of normal and mutant clones from the SMCD patient. The sequence shown is from nucleotide 1984 to 1998 of COLIOAI and the position of the mutation is indicated.

than the wild type polypeptide (680 amino acids). Neither of the parents had this mutation. A previously reported polymorphic amino acid substitution (Gly545Arg) (Sweetman et al., 1992) was detected in the patient and her father, but not in her mother (data not shown).

All of the known SMCD mutations are different (Warman et al., 1993; Wallis et al., 1994; McIntosh et al., 1994, 1995; Dharmavaram et al., 1994; Chan et al., 1995; Bonaventure et al., 1995). However, three of the reported deletions were initiated at nucleotide 1856 of COL1OA1. Thus, it seems that this nucleotide may 
be more prone to mutational events. A 1992delCT mutation of COL10Al has been previously reported in one family (McIntosh et al., 1994). The data presented here indicate that an apparently unrelated Japanese SMCD patient has a recurrent 1992delCT mutation of COL10A1. This dinucleotide locates within the direct repeat sequence (CTCT at nt 1992-1995), and the gene deletions have been suggested to occur preferentially at such sites (Krawczak and Cooper, 1991). The independent occurrence of de novo mutation in this specific dinucleotide repeat suggests that this region is a possible mutational hot spot on COL10A1. Although some SMCD patients are reported to have no mutation of COL10A1 (Bonaventure et al., 1995), these observations indicate allelic heterogeneity and confirm the role of mutation within the C-terminal noncollagenous domain coding region of COL1OAl in the pathogenesis of most SMCD.

Acknowledgments This work was supported in part by Scientific Research Grant 06454428 from the Ministry of Education, Science and Culture of Japan.

\section{REFERENCES}

Bonaventure J, Chaminade F, Maroteaux $P$ (1995): Mutations in three subdomains of the carboxy-terminal region of collagen type $X$ account for most of the Schmid metaphyseal dysplasias. Hum Genet 96: 58-64

Chan D, Cole WG, Rogers JG, Bateman JF (1995): Type X collagen multimer assembly in vitro is prevented by a Gly ${ }^{618}$ to Val mutation in the $\alpha 1(\mathrm{X}) \mathrm{NCl}$ domain resulting in Schmid metaphyseal chondrodysplasia. J Biol Chem 270: 4558-4562

Dharmavaram RM, Elberson MA, Peng M, Kirson LA, Kelley TE, Jimenez SA (1994): Identification of a mutation in type $X$ collagen in a family with Schmid metaphyseal chondrodysplasia. Hum Mol Genet 3: 507-509

Krawczak M, Cooper DN (1991): Gene deletions causing human genetic disease: mechanisms of mutagenesis and the role of the local DNA sequence environment. Hum Genet 86: 425-441

McIntosh I, Abbott MH, Warman ML, Olsen BR, Francomano CA (1994): Additional mutations of type $\mathrm{X}$ collagen confirm COL10Al as the Schmid metaphyseal chondrodysplasia locus. Hum Mol Genet 3: 303-307

McIntosh I, Abbott MH, Francomano CA (1995): Concentration of mutations causing Schmid metaphyseal chondrodysplasia in the $\mathrm{C}$-terminal noncollagenous domain of type $\mathrm{X}$ collagen. Hum Mutat 5: 121-125

Sambrook J, Fritsch EF, Maniatis T (1989): Molecular cloning: a laboratory manual, 2nd ed. Cold Spring Harbor Laboratory Press, New York

Sweetman WA, Rash B, Sykes B, Beighton P, Hecht JT, Zabel B, Thomas JT, Boot-Handford R, Grant ME, Wallis GA (1992): SSCP and segregation analysis of the human type X collagen gene (COL10A1) in heritable forms of chondrodysplasia. Am J Hum Genet 51: 841-849

Wallis GA, Rash B, Sweetman WA, Thomas JT, Super M, Evans G, Grant ME, Boot-Handford RP (1994): Amino acid substitutions of conserved residues in the carboxyl-terminal domain of the $\alpha \mathrm{l}(\mathrm{X})$ chain of type $\mathrm{X}$ collagen occur in two unrelated families with metaphyseal chondrodysplasia type Schmid. Am J Hum Genet 54: 169-178

Warman ML, Abbott M, Apte SS, Hefferon T, McIntosh I, Cohn DH, Hecht JT, Olsen BR, Francomano CA (1993): A type X collagen mutation causes Schmid metaphyseal chondrodysplasia. Nature Genet 5: 79-82 